\title{
Immune Checkpoint Inhibitors for Esophageal Cancer
}

\author{
Toshiharu Hirose and Shun Yamamoto* \\ Department of Head and Neck Medical Oncology, National Cancer Center Hospital, Japan
}

\begin{abstract}
Esophageal cancer (EC) is the seventh most common cancer, and patients with advanced EC have a poor prognosis. Recently, nivolumab and pembrolizumab, two immune checkpoint inhibitors (ICIs) that inhibit programmed cell death protein 1, have begun to be used for the treatment of advanced esophageal squamous cell carcinoma (ESCC). Based on the ATTRACTION-3 and KEYNOTE-181 trials, the use of nivolumab and pembrolizumab (only in programmed cell death ligand 1-positive cases) was approved for treatment by the US Federal Drug Administration for the treatment of patients with advanced ESCC. In addition, the CheckMate-577 and the KEYNOTE-590 trials have shown survival benefits for postoperative nivolumab in patients with resectable EC and for first line pembrolizumab plus doublet chemotherapy in patients with advanced EC, respectively. Many trials to evaluate ICI-containing treatments are ongoing, and these new treatments are expected to improve the clinical outcomes of patients with EC.
\end{abstract}

KEYWORDS: Esophageal cancer; Esophageal squamous cell carcinoma; Immune checkpoint inhibitor; Nivolumab; Pembrolizumab

ABBREVIATIONS: EC: Esophageal Cancer; ESCC: Esophageal Squamous Cell Carcinoma; ICI: Immune Checkpoint Inhibitors; EAC: Esophageal Adenocarcinoma; PD-1: Programmed Cell Death1; PD-L1: Programmed Death Ligand1; ORR: Objective Response Rate; OS: Overall Survival; PFS: Progression Free Survival; FDA: Food and Drug Administration; EGJ: Esophagogastric Junction; DFS: Disease Free Survival

\section{INTRODUCTION}

Esophageal cancer (EC) is the seventh most common cancer and the sixth leading cause of cancer deaths worldwide [1]. There are two main histological subtypes of EC: esophageal squamous cell carcinoma (ESCC), and esophageal adenocarcinoma (EAC). ESCC is the most common subtype globally, and the incidence of ESCC is especially high in Eastern Asia and Eastern Africa. On the other hand, the incidence of EAC is especially high in Western countries [2-5]. EC is generally an extremely aggressive cancer. Patients with early-stage EC are often asymptomatic, and many patients are diagnosed at an advanced stage after the appearance of symptoms $[5,6]$. In addition, effective chemotherapeutic drugs for metastatic or recurrent EC are limited. Systemic chemotherapy is the standard treatment for patients with metastatic or recurrent EC who have no curative treatment options. Systemic chemotherapy is aimed at controlling cancer-related symptoms and prolonging survival [7-9].
No randomized trials have shown the survival benefits of palliative chemotherapy other than best-supportive care as a first-line treatment, and doublet chemotherapy consisting of platinum and fluoropyrimidine is recognized as a standard firstline chemotherapy for patients with advanced EC [7-11]. For a long time, salvage-line chemotherapy after refractoriness or intolerance to first-line chemotherapy consisted of taxane monotherapy $[12,13]$ or irinotecan monotherapy [7].

\section{IMMUNE CHECKPOINT INHIBITORS}

Immune checkpoint inhibitors (ICIs) targeting the programmed cell death 1 (PD-1)/ programmed death-ligand 1 (PD-L1) pathway have shown a survival benefit when used against many cancer types. Inhibitory immune checkpoint molecules and stimulatory immune checkpoint molecules play an important role in the maintenance of immunological homeostasis. The dysregulation

\begin{tabular}{|c|c|}
\hline \multirow{2}{*}{$\begin{array}{c}\text { Quick Response Code: } \\
\text { (a) }\end{array}$} & $\begin{array}{l}\text { Address for correspondence: Shun Yamamoto, Dep } \\
\text { Medical Oncology, National Cancer Center Hospital, Jap }\end{array}$ \\
\hline & $\begin{array}{l}\text { Published: March 26, } 2021 \\
\text { Iow to cite this article: Toshiharu H, Shun Y. Immune Checkpoint Inhibitors for Esophageal } \\
\text { ancer. 2021- 3(2) OAJBS.ID.000267. DOI: 10.38125/OAJBS.000267 }\end{array}$ \\
\hline
\end{tabular}


of inhibitory immune checkpoint molecules expression by cancer cells is associated with escape from immune surveillance, which is a key mechanism driving tumor progression.

PD-1 is an immunosuppressive receptor that is highly expressed on immune cells, such as activated lymphocytes [14]. The interaction between PD-1 and PD-L1 or 2 could mediate the suppression of T cell activity through the negative regulation of $\mathrm{T}$ cell receptors and CD28 signaling [14]. PD-L1 overexpression is prevalent in many cell types, including antigen-presenting cells [15]. The upregulation of PD-L1 is also found in many types of cancer, suggesting that the PD-1/PD-L1 pathway impairs the antitumor response [14]. PD-L1 overexpression is observed in $18.4 \%-82.8 \%$ of ESCC patients, and these patients showed poor clinical outcomes [16].

\section{NIVOLUMAB}

\section{Attraction-1/Attraction-3 Trial}

The ATTRACTION-1 phase II trial was conducted to evaluate the efficacy and safety of nivolumab monotherapy $(3 \mathrm{mg} / \mathrm{kg}$, every 2 weeks) after the failure of fluoropyrimidine-based, platinum-based, and taxane-based chemotherapies, regardless of PD-L1 expression, in patients with advanced EC. Sixty-five patients were included, and nivolumab showed a promising anti-tumor efficacy with an objective response rate (ORR) of 11 patients (17\%, 95\% CI: $10 \%$ $28 \%$ ), including 3 patients who achieved a complete response. The median overall survival (OS) was 10.78 months (95\% CI: 7.4-13.3 months), and the median progression-free survival (PFS) was 1.5 months (95\% CI: 1.4-2.8 months). The most frequent grade 3-4 adverse events were lung infection (3\%) and dehydration (3\%), and no deaths related to nivolumab occurred [17].

Based on the results of the ATTRACTION-1 trial, the ATTRACTION-3 phase III trial was conducted to verify the superior efficacy of nivolumab monotherapy ( $240 \mathrm{mg} /$ body, every 2 weeks) compared with taxanes (paclitaxel or docetaxel) in patients with advanced ESCC who refractory to fluoropyrimidine and platinum treatments were, regardless of PD-L1 expression. Nivolumab demonstrated a significant improvement in OS, compared with the taxane arm (median OS: 10.9 vs. 8.4 months, HR [95\% CI]: 0.77 [0.62-0.96], $p=0.019$ ). The ORR was $19 \%$ (95\% CI: $14 \%-26 \%)$ in the nivolumab arm and 22\% (95\% CI: $15 \%-29 \%)$ in the taxane arm. In the nivolumab arm, the most frequent serious adverse events were pyrexia (2\%) and intestinal lung disease (2\%), and two deaths related to the use of nivolumab occurred (interstitial lung disease and pneumonitis). Based on the results of the ATTRACTION-3 trial, the US Food and Drug Administration (FDA) approved nivolumab monotherapy as a treatment for patients with advanced ESCC after prior fluoropyrimidine- and platinum-based chemotherapy, regardless of PD-L1 expression, on June 10, 2020. Nivolumab has since become the standard second-line chemotherapy for patients with advanced ESCC.

\section{CHECKMATE-577 TRIAL}

The CheckMate-577 phase III trial was conducted to compare nivolumab monotherapy to a placebo as a postoperative treatment after the complete resection of resectable EC or esophagogastric junction (EGJ) cancer in patients who had received preoperative chemoradiotherapy and in whom a pathologic complete response was not achieved. In this trial, 794 patients were randomly assigned to a nivolumab group $(\mathrm{n}=532 ; 240 \mathrm{mg} /$ body, every 2 weeks for 16 weeks, followed by nivolumab $480 \mathrm{mg} /$ body, every 4 weeks) or a placebo group ( $\mathrm{n}=262)$. A pre-specified interim analysis showed that postoperative nivolumab enabled a statistically significant improvement in disease-free survival (DFS) compared with the placebo (median DFS: 22.4 months [95\% CI: 6.6-34.0 months] vs. 11.0 months [95\% CI: 8.3-14.3 months], HR [95\% CI]: 0.69 [0.560.86 ], $p=0.0003$ ). Immune-related grade 3 or higher adverse events were reported in $7 \%$ of patients in the nivolumab arm $[18,19]$.

\section{PEMBROLIZUMAB}

\section{Keynote-180/Keynote-181 Trial}

The KEYNOTE-180 phase II trial was conducted to evaluate the safety and efficacy of pembrolizumab according to histology and PD-L1 positivity in heavily treated patients with metastatic EC patients [20]. The ORR was 9.9\% (95\% CI: 5.2\%-16.7\%) in the total population. The ORRs of subgroups with ESCC, EAC, PD-L1positive tumors, and PD-L1-negative tumors were 14.3\%, 5.2\%, $13.8 \%$, and $6.3 \%$, respectively. The median PFS was 2.0 months (95\% CI: 1.9-2.1 months) in all the patients. The median OS was 5.8 months (95\% CI: 4.5-7.2 months) in all the patients. The OS periods of the subgroups with ESCC, EAC, PD-L1-positive tumors, and PDL1-negative tumors were 6.8 months, 5.4 months, 3.9 months, and 5.4 months, respectively. These results supported pembrolizumab exhibiting a meaningful antitumor activity in heavily treated EC regardless of histology or PD-L1 expression. The safety profile was consistent with that of pembrolizumab in previous studies.

Sequentially, the KEYNOTE-181 phase III trial was conducted to compare pembrolizumab with the investigator's choice of chemotherapy (paclitaxel, docetaxel, or irinotecan) as a secondline treatment in patients who had advanced EC. The median OS of 9.3 months in the pembrolizumab arm was prolonged compared with an OS of 6.7 months in the chemotherapy arm (HR [95\% CI]: 0.69 [0.52-0.93], $p=0.0074$ ) in EC patients with a PD-L1 combined positive score (CPS) $\geq 10$. However, pembrolizumab did not enable a superior OS compared with chemotherapy in patients with ESCC (median OS: 8.2 months vs. 7.1 months, HR [95\% CI]: 0.78 [0.63$0.96], p=0.0095$ ) or overall (median OS: 7.1 months vs. 7.1 months, HR [95\% CI]: 0.89 [0.75-1.05], $p=0.0560)$. The ORRs of the EC patients with a PD-L1 CPS $\geq 10$ were $21.5 \%$ vs. $6.1 \%$ ( $p=0.0006)$. The ORRs of the ESCC patients were $16.7 \%$ vs. $7.4 \%(p=0.0022)$ [21]. Based on these results, on July 30, 2019, the FDA approved pembrolizumab as a second-line chemotherapy for patients with advanced or metastatic ESCC and a PD-L1 CPS $\geq 10$.

\section{KEYNOTE-590 TRIAL}

The KEYNOTE-590 phase III trial was conducted to compare pembrolizumab (200 mg every 3 weeks for $\leq 35$ cycles) plus chemotherapy (5-FU $800 \mathrm{mg} / \mathrm{m}^{2}$ for days 1-5 every 3 weeks for $\leq 35$ cycles + cisplatin $80 \mathrm{mg} / \mathrm{m}^{2}$ every 3 weeks for $\leq 6$ cycles) with chemotherapy alone (placebo+ 5-FU $800 \mathrm{mg} / \mathrm{m}^{2}$ for days $1-5$ every 3 weeks for $\leq 35$ cycles + cisplatin $80 \mathrm{mg} / \mathrm{m}^{2}$ every 3 weeks for $\leq 6$ cycles) for patients with advanced EC. In patients with ESCC, the median OS periods in the pembrolizumab plus chemotherapy and chemotherapy alone arms were 12.6 and 9.8 months, respectively (HR [95\% CI]: 0.72 [0.6-0.88], $p<0.0006$ ); overall, the median OS periods in the pembrolizumab plus chemotherapy and chemotherapy alone arms were 12.4 months (95\% CI: $10.5-14.0$ ) and 9.8 months (95\% CI: 8.8-10.8), respectively (HR [95\% CI]: 0.73 [0.62-0.86], $p<0.0001)$. In patients with ESCC, the median PFS periods were 6.3 months (95\% CI: 6.2-6.9) and 5.8 months (95\% CI: 5.0-6.1), respectively (HR [95\% CI]: 0.65 [0.54-0.78], $p<$ $0.0001)$; overall, the median PFS periods were 6.3 months (95\% CI: 
6.2-6.9) and 5.8 months (95\% CI: 5.0-6.0), respectively (HR [95\% CI]: 0.65 [0.55-0.76], $p<0.0001)$. The secondary endpoint of ORR was also significantly improved by the addition of pembrolizumab (45\% [95\% CI: 39.9-50.2] vs. 29.3\% [95\% CI: 24.7-34.1], $p<$
0.0001) [22]. The results of the KEYNOTE-590 trial suggested that pembrolizumab plus doublet chemotherapy could be a new standard first-line treatment (Table 1).

Table 1: Pivotal clinical trials for ICls in patients with EC.

\begin{tabular}{|c|c|c|c|c|c|c|c|c|}
\hline Clinical Trial & Phase & Histology & Line & No. of Patients & Regimen & ORR (\%) & PFS (m) & OS (m) \\
\hline \multicolumn{9}{|c|}{ Nivolumab } \\
\hline ATTRACTION-1 & II & ESCC & $3^{\text {rd }}$ or later & 65 & Nivolumab & 30 & 1.8 & 7 \\
\hline ATRRACTION-3 & III & ESCC & $2^{\text {nd }}$ & 419 & Nivolumab vs. taxane & 9.9 & 2 & 5.8 \\
\hline CheckMate-577 & III & ESCC, EAC & Post-operative & 794 & $\begin{array}{l}\text { Postoperative nivolumab } \\
\text { vs. placebo }\end{array}$ & NA & $22.4^{*}$ & NA \\
\hline \multicolumn{9}{|c|}{ Pembrolizumab } \\
\hline KEYNOTE-180 & II & ESCC, EAC & $3^{\text {rd }}$ or later & 121 & Pembrolizumab & 30 & 1.8 & 7 \\
\hline KEYNOTE-181 & II & ESCC, EAC & $2^{\text {nd }}$ & 628 & $\begin{array}{c}\text { Pembrolizumab vs. } \\
\text { cytotoxic chemotherapy }\end{array}$ & 13.1 & 2.1 & 7.1 \\
\hline KEYNOTE-590 & III & ESCC, EAC & $1^{\text {st }}$ & 749 & $\begin{array}{l}\text { Pembrolizumab + CF vs. } \\
\text { Placebo + CF }\end{array}$ & 45 & 6.3 & 12.4 \\
\hline
\end{tabular}

*Disease-free survival

Abbreviations: ORR: Objective Response Rate; PFS: Progression-Free Survival; OS: Overall Survival; CF: cisplatin + 5-FU; NA: Not Assessed.

\section{ONGOING TRIALS}

A number of clinical trials are being conducted using ICIs in combination with existing treatments. Some of the key trials are presented below. For untreated advanced ESCC patients, the CheckMate-648 phase III trial (NCT03143153) comparing the efficacy of nivolumab plus doublet chemotherapy or nivolumab plus ipilimumab with doublet chemotherapy as a first-line treatment has finished recruitment and is awaiting analysis [23].

For locally advanced EC patients, the KEYNOTE-975 phase III trial (NCT04210115) comparing definitive chemoradiotherapy plus pembrolizumab with the standard definitive chemoradiotherapy is presently recruiting patients.

Additionally, a phase I trial based on preoperative chemotherapy, the JCOG1804E (FRONTiER) trial (NCT03914443), will evaluate the safety and efficacy of nivolumab plus 5-FU and cisplatin or nivolumab plus docetaxel, 5-FU, and cisplatin for resectable locally advanced ESCC patients; this trial is ongoing [24].

\section{DISCUSSION}

ICIs have become the second-line standard treatment mainly for patients with advanced ESCC, and about half of patients exhibit progressive disease at the time of their first imaging evaluation. Therefore, useful biomarkers for ICI treatment are needed. For example, high microsatellite instability or a high tumor mutational burden are important biomarkers suggesting the efficacy of ICIs, but these patient populations are relatively small $[25,26]$. For lung cancer patients, PD-L1 expression has been reported as an important biomarker for nivolumab and pembrolizumab, and the ATTRACTION-3 and KEYNOTE-181 trials evaluated the clinical benefits of nivolumab and pembrolizumab, respectively, in patients with PD-L1 expression. In the ATTRACTION-3 trial, PD-L1 expression was evaluated using the tumor proportion score (TPS) (only tumor cells, using 28-8 antibody); as a result, PD-L1 expression was shown to have no effect on the survival benefits of nivolumab. On the other hand, in the KEYNOTE-181 trial, PD-L1 expression was evaluated using the CPS (tumor cell and lymphocytes, macrophages, using 22C3 antibody), and PD-L1 expression was suggested to affect the efficacy (OS) of pembrolizumab.

Given the data from the BLUEPRINT project, differences in the types of cells that are evaluated for PD-L1 expression might have a greater effect on study results than differences in the types of antibodies that are used [27]. Therefore, we expect that TPS and CPS are different biomarkers, but the concordance between TPS and CPS has not yet been evaluated in EC patients. Further investigation is therefore needed.

\section{CONCLUSION}

The ATTRACTION-3 and KEYNOTE0181 trials created a new epoch in the use of ICIs for the treatment of advanced EC. In addition, the CheckMate- 577 trial showed a survival benefit for postoperative nivolumab in a resectable setting, and the KEYNOTE-590 trial showed a survival benefit of first-line pembrolizumab plus doublet chemotherapy in an advanced setting. Additionally, some trials to evaluate ICI-containing investigational treatments are ongoing, and these results might further improve the survival outcomes for patients with EC.

\section{CONFLICT OF INTEREST}

TH has nothing to declare. SY has received honoraria from ONO Pharmaceuticals and Bristol-Myers Squibb.

\section{REFERENCES}

1. World Health Organization (2020) GLOBOCAN 2020 Estimated cancer incidence, mortality and prevalence worldwide.

2. Abnet CC, Arnold M, Wei WQ (2018) Epidemiology of esophageal squamous cell carcinoma. Gastroenterology 154(2): 360-373.

3. Njei B, McCarty TR, Birk JW (2016) Trends in esophageal cancer survival in United States adults from 1973 to 2009: A SEER database analysis. J Gastroenterol Hepatol 31: 1141-1146. 
4. Torre LA, Siegel RL, Ward EM, Jemal A (2016) Global cancer incidence and mortality rates and trends-an update. Cancer Epidemiol Biomarkers Prev 25: 16-27.

5. Murphy G, McCormack V, Abedi-Ardekani B, Arnold M, Dar NA, et al. (2017) International cancer seminars: A focus on esophageal squamous cell carcinoma. Ann Oncol 28: 2086-2093.

6. Rustgi AK, El- Serag HB (2014) Esophageal carcinoma. N Engl J Med: 371: 2499-2509.

7. NCCN guidelines v (2020) Esophageal and esophagogastric junction cancers.

8. Lordick F, Mariette C, Haustermans K, Obermannova R, Arnold D, et al. (2016) Oesophageal cancer: ESMO Clinical Practice Guidelines for diagnosis, treatment and follow-up. Ann Oncol 27 (suppl 5): v50-v57.

9. Kitagawa Y, Uno T, Oyama T, Kato K, Kato H, et al. (2019) Esophageal cancer practice guidelines 2017 edited by the Japan esophageal society: part 2. Esophagus 16(1): 25-43.

10. Iizuka T, Kakegawa T, Ide H, Ando N, Watanabe H, et al. (1992) Phase II evaluation of cisplatin and 5-fluorouracil in advanced squamous cell carcinoma of the esophagus: a Japanese Esophageal Oncology Group Trial. Jpn J Clin Oncol 22(3): 172-176.

11. Hayashi K, Ando N, Watanabe H, Ide H, Aoyama N, et al. (2001) Phase II evaluation of protracted infusion of cisplatin and 5-fluorouracil in advanced squamous cell carcinoma of the esophagus: a Japan Esophageal Oncology Group. Jpn J Clin Oncol: 31(9), 419-423.

12. Kato K, Tahara M, Hironaka S, Muro K, Takiuchi H, et al. (2011) A phase II study of paclitaxel by weekly 1-h infusion for advanced or recurrent esophageal cancer in patients who had previously received platinumbased chemotherapy. Cancer Chemother Pharmacol 67(6): 1265-1272.

13. Muro K, Hamaguchi T, Ohtsu A, Boku N, Chin K, et al. (2004) A phase Il study of single-agent docetaxel in patients with metastatic esophageal cancer. Ann Oncol 15(6): 955-959.

14. Sharpe AH, Pauken KE (2018) The diverse functions of the PD1 inhibitory pathway. Nat Rev Immunol 18(3): 153-167.

15. Boussiotis VA (2016) Molecular and biochemical aspects of the PD-1 checkpoint pathway. N Engl J Med 375(18): 1767-1778.

16. Guo W, Wang P, Li N, Shao F, Zhang H, et al. (2017) Prognostic value of PDL1 in esophageal squamous cell carcinoma: a meta-analysis. Oncotarget 9(17): 13920-13933.

17. Kudo T, Hamamoto Y, Kato K, Ura T, Kojima T, et al. (2017) Nivolumab treatment for oesophageal squamous-cell carcinoma: an open-label, multicentre, phase 2 trial. Lancet Oncol 18(5): 631-639.
18. Kato K, Cho BC, Takahashi M, Okada M, Lin CY, et al. (2019) Nivolumab versus chemotherapy in patients with advanced oesophageal squamous cell carcinoma refractory or intolerant to previous chemotherapy (ATTRACTION-3): A multicentre, randomised, open-label, phase 3 trial. Lancet Oncol 20(11): 1506-1517.

19. Kelly RJ, Ajani JA, Kuzdzai J, Zander T, Van Cutsem E, et al. (2020) Adjuvant nivolumab in resected esophageal or gastroesophageal junction cancer (EC/GEJC) following neoadjuvant chemoradiation therapy (CRT): first results of the CheckMate 577 study. Presented at ESMO Virtual Congress.

20. Shah MA, Kojima T, Hochhauser D, Enzinger P, Raimbourg J, et al. (2019) Efficacy and safety of pembrolizumab for heavily pretreated patients with advanced, metastatic adenocarcinoma or squamous cell Carcinoma of the esophagus: the phase 2 KEYNOTE-180 study. JAMA Oncol 5(4): 546-550.

21. Kojima T, Muro K, Francois E, Hsu CH, Moriwaki T, et al. (2019) Pembrolizumab versus chemotherapy as second-line therapy for advanced esophageal cancer: phase III KEYNOTE-181 study. J Clin Oncol 37(4_suppl): 2 .

22. Kato K, Sun JM, Shah MA (2020) Pembrolizumab plus chemotherapy versus chemotherapy as first-line therapy in patients with advanced esophageal cancer: The phase 3 KEYNOTE-590 study. Presented at ESMO Virtual Congress.

23. Ajani JA, Kato K, Doki Y, Chau I, Xynos I, et al. (2018) CheckMate 648: a randomized phase 3 study of nivolumab plus ipilimumab or nivolumab combined with fluorouracil plus cisplatin versus fluorouracil plus cisplatin in patients with unresectable advanced, recurrent, or metastatic previously untreated esophageal squamous cell carcinoma. J Clin Oncol 36(4): TPS193.

24. Yamamoto S, Kato K, Daiko H, Kojima T, Hara H, et al. (2017) Feasibility study of nivolumab as neoadjuvant chemotherapy for locally esophageal carcinoma: FRONTiER (JCOG1804E). Future Oncol 16(19): 1351-1357.

25. Le DT, Durham JN, Smith KN, Wang H, Bartlett BR, et al. (2017) Mismatch repair deficiency predicts response of solid tumors to PD-1 blockade. Science 357(6349): 409-413.

26. Marabelle A, Fakih M, Lopez I, Shah M, Shapira-Frommer R, et al. (2020) Association of tumour mutational burden with outcomes in patients with advanced solid tumours treated with pembrolizumab: prospective biomarker analysis of the multicohort, open-label, phase 2 KEYNOTE-158 study. Lancet Oncol 21(10): 1353-1365.

27. Tsao MS, Kerr KM, Kockx M, Beasley MB, Borczuk AC, et al. (2018) PD-L1 immunohistochemistry comparability study in real-life clinical samples: Results of Blueprint Phase 2 Project. J Thorac Oncol 13(9): 1302-1311. 